\title{
Sarocladium spinificis, a new endophytic species from the coastal grass Spinifex littoreus in Taiwan
}

\author{
Yu-Hung Yeh and Roland Kirschner*
}

\begin{abstract}
Background: Sarocladium species are frequently associated with grasses as saprobes, parasites, and mutualistic endophytes. A species of Sarocladium (anamorphic Hypocreales) was isolated as endophytic fungus from the coastal grass Spinifex littoreus (Poaceae).

Results: According to characterization by LSU and ITS rDNA sequences and culture morphology and micromorphology, the species differed from the species hitherto described in Sarocladium. A key to the known species of Sarocladium is given.

Conclusions: Sarocladium spinificis is proposed as a new species. LSU rDNA sequences and conidiophore branching and conidium size are useful characters for distinguishing between species of Sarocladium.
\end{abstract}

Keywords: Ascomycota; Coastal dunes; Endophytes; Sandy beach; Taxonomy

\section{Background}

The genus Sarocladium was erected by Gams and Hawksworth (1976) based on a species described by Sawada in Taiwan, first including two fungal pathogens causing sheath rot of rice, with the type species Sarocladium oryzae (Sawada) W. Gams \& D. Hawksw., and S. attenuatum W. Gams \& D. Hawksw. (Gams and Hawksworth, 1976). The characteristics of the genus are phialides being narrowly cylindrical, hardly tapering towards the apices, lacking collarettes, producing onecelled, cylindrical, hyaline conidia (Gams and Hawksworth, 1976). Sarocladium sinense J.D. Chen, Guo C. Zhang \& X. H. Fu and S. mycophilum Helfer were described in 1987 and 1991, respectively (Helfer, 1991; Liao and Wu, 2000). In 2011, according to phylogenetic analysis based on sequences of the LSU rDNA, Sarocladium was extended to include 7 species formerly placed in Acremonium (Summerbell et al., 2011). By molecular analysis, Sarocladium can be connected to the Hypocreales, but the relationship with a teleomorph on the species, genus, and family level is completely unknown. The species of Sarocladium have many important applications. For example, S. oryzae produces antibiotics, S. kiliense (Grütz) Summerb. can cause human diseases, $S$. strictum is able to cause

\footnotetext{
* Correspondence: Kirschner@ncu.edu.tw

Department of Life Sciences, National Central University, No. 300, Jhongda Road, Jhongli City, Taoyuan County 32001, Taiwan (R.O.C.)
}

disease of sorghum and strawberry plants, and S. zeae (W. Gams \& D.R. Sumner) Summerb. is considered a mutualistic maize endophyte protective against herbivory (Tschen et al., 1997; Bills et al., 2004; Summerbell et al., 2011; Racedo et al., 2013, as Acremonium strictum). Most strains have been isolated from members of Poaceae, such as bamboo, maize, rice, and other cereals and wild grasses (Gams, 1971; Gams and Hawksworth, 1976; Summerbell et al., 2011).

On the coast of Taiwan, the grass Spinifex littoreus (Burm. f.) Merr. (Poaceae) grows in littoral sandy beaches and dunes. To propagate and over-come sand burial, the species produces vertical and horizontal rhizomes and stolons. By this growth, populations of this plant contribute to the formation of dunes (Maun, 2009). Though Sp. littoreus is important for stabilizing and protecting coastal areas, very little has been published about the organisms associated with this plant. For this reason, we studied endophytes and other fungi associated with this plant. Among the fungi most frequently isolated as endophytes, a species was identified as member of Sarocladium, but was not identical to any of the previously described species.

\section{Methods}

Plants and collection sites

During the years 2011-2012, individuals of Spinifex littoreus were collected in seven locations distributed on 
sandy sites of the northern, western and southern coast of Taiwan. Plants were collected from five beaches of five counties in Taiwan (Yilan $24.718^{\circ} \mathrm{N}, 121.832^{\circ} \mathrm{E}$, Taoyuan $25.047^{\circ} \mathrm{N}, 121.076^{\circ} \mathrm{E}$, Hsinchu $24.765^{\circ} \mathrm{N}, 120.911^{\circ} \mathrm{E}$, Miaoli $24.622^{\circ} \mathrm{N}, 120.756^{\circ} \mathrm{E}$ and Taipei $25.029^{\circ} \mathrm{N}, 121.935^{\circ} \mathrm{E}$ ), while in the two remaining counties (Pingtung and Chiayi), plants were obtained at other habitats. In Pingtung County, plants were collected at Hengchun Tropical Botanic Garden $\left(21.958^{\circ} \mathrm{N}, 120.811^{\circ} \mathrm{E}\right), 200-300 \mathrm{~m}$ above sea level, at a distance of $2 \mathrm{~km}$ from the coast. In Chiayi, the site was coastal wetland close to Haomeiliao $\left(23.360^{\circ} \mathrm{N}, 120.129^{\circ} \mathrm{E}\right)$. The plants were processed for endophyte isolation within 72 hours after sampling.

\section{Isolation of fungi}

Plants were removed with a trowel, individually placed in bags, returned to the laboratory and kept at $4^{\circ} \mathrm{C}$ until further processing. Samples were washed and divided into root, stem, leaf sheath and leaf lamina. All healthy plant parts from Spinifex littoreus were cut transversally into three fragments. Furthermore, seeds of Sp. littoreus were used. Plant fragments were surface-sterilized by agitation in $95 \%$ ethanol for $1 \mathrm{~min}, 6-12 \%$ sodium hypochlorite for $3 \mathrm{~min}$, 95\% ethanol for $0.5 \mathrm{~min}$, and then rinsed in sterile water, and placed onto malt extract agar (MEA). The effectiveness of surface sterilization was tested by making imprints of plant fragments on malt extract agar plates (Rodriguez et al., 2008). All isolates obtained from each plant sample were classified according to their morphological appearance into morphotypes. Representative isolates of a species of Sarocladium were investigated and deposited at the Bioresource and Collection Center, Hsinchu, Taiwan (BCRC). A dried culture was deposited at the herbarium of the National Museum of Natural Science, Taichung, Taiwan (TNM).

\section{DNA isolation and molecular analysis}

DNA was extracted from all examined strains with Genomic DNA Spin Kit (Plant), according to the modified manufacturer's protocol (Bioman Scientific Co., Ltd., Taiwan). Primer pairs ITS1F/ITS4 and NL1/NL4 were used for amplification of the ITS and partial LSU rDNA, respectively (Gardes and Bruns, 1993; White et al., 1990). Success of the amplification was assessed with $2 \%$ agarose gel electrophoresis followed by staining with GelRed ${ }^{\text {tw }}$ (Biotium, Hayward, California, U.S.A.) visualized under UV light (312 nm). Illustra GFX PCR DNA and Gel Band Purification Kit, GE Healthcare, UK, were applied for purification of the PCR products. Sequencing of DNA was done by Mission Biotech (Nankang, Taipei) with the same primers as for the PCR. DNA sequences were edited using CodonCode Aligner version 4.0.1 (CodonCode Corporation, USA). Related DNA sequences of ITS and LSU rDNA were compared using the BLAST function of
GenBank. For phylogenetic analysis, sequences retrieved from the BLAST search and the taxon sampling based on LSU rDNA sequences in Summerbell et al. (2011) were used. In addition to sequences of Sarocladium spp., those of Acremonium species closely related with Sarocladium spp., but outside the Sarocladium clade, were included as outgroup (Summerbell et al., 2011). Altogether 27 nucleotide sequences were aligned using the default options of MUSCLE implemented in MEGA5 (Tamura et al., 2011) without manual editing except for truncating the ends so that a block of 481 positions in the final dataset was obtained. The evolutionary history was inferred by using the Maximum Likelihood method with the default options of MEGA5, based on the Tamura-Nei model and 1000 bootstrap replications. The tree shown in Figure 1 was not rooted.

\section{Morphological study}

Fungal growth rates were evaluated after transfer of conidia with a flamed needle onto three plates containing $2 \%$ maltextract agar (MEA) with $0.2 \%$ chloramphenicol and incubation at $25^{\circ} \mathrm{C}$. After 10 days, the diameter of the colony was measured. For evaluating the culture morphology, corn meal agar (CMA) and potato dextrose agar (PDA) were also used. Online Auction Color Chart ${ }^{\circ}$ was used as reference for color terms. Microscopic characteristics were observed using fungal material mounted in $5-10 \%(\mathrm{w} / \mathrm{v})$ aqueous $\mathrm{KOH}$ solution and $1 \%$ phloxine. Statistical treatment of measurements was based on 30 measurements of the phialides of the designated ex-type strain which are given as mean value \pm standard deviation with extreme values given in brackets. The same method was used for 30 conidia per each of five strains, i.e. altogether 150 conidia.

\section{Results}

Among 1670 isolates, 307 (ca. 18\%) were identified as Sarocladium sp. An analysis of all endophytes will be presented in another publication. As shown in Figure 1, the isolates from Spinifex littoreus (GenBank accession numbers of newly created LSU rDNA sequences JQ954465, JQ954463, KC920827, JQ954466, KC920828, KC920829) form a strongly supported clade which is significantly separated from sequences of other Sarocladium species. A sequence of the ITS (594 bp) was deposited in GenBank as KF269096. When comparing the BLAST search results among sequences exceeding a length of 539 bp of ITS rDNA fragments, the highest similarity between our isolates and other identified Sarocladium species was $95 \%$, namely with one strain of Sarocladium strictum (Genbank number AY428790, Saleh and Leslie, 2004) showing a similarity of $94 \%-$ $95 \%$. According to morphology, the new species is most similar to Sarocladium strictum, because S. strictum is a 


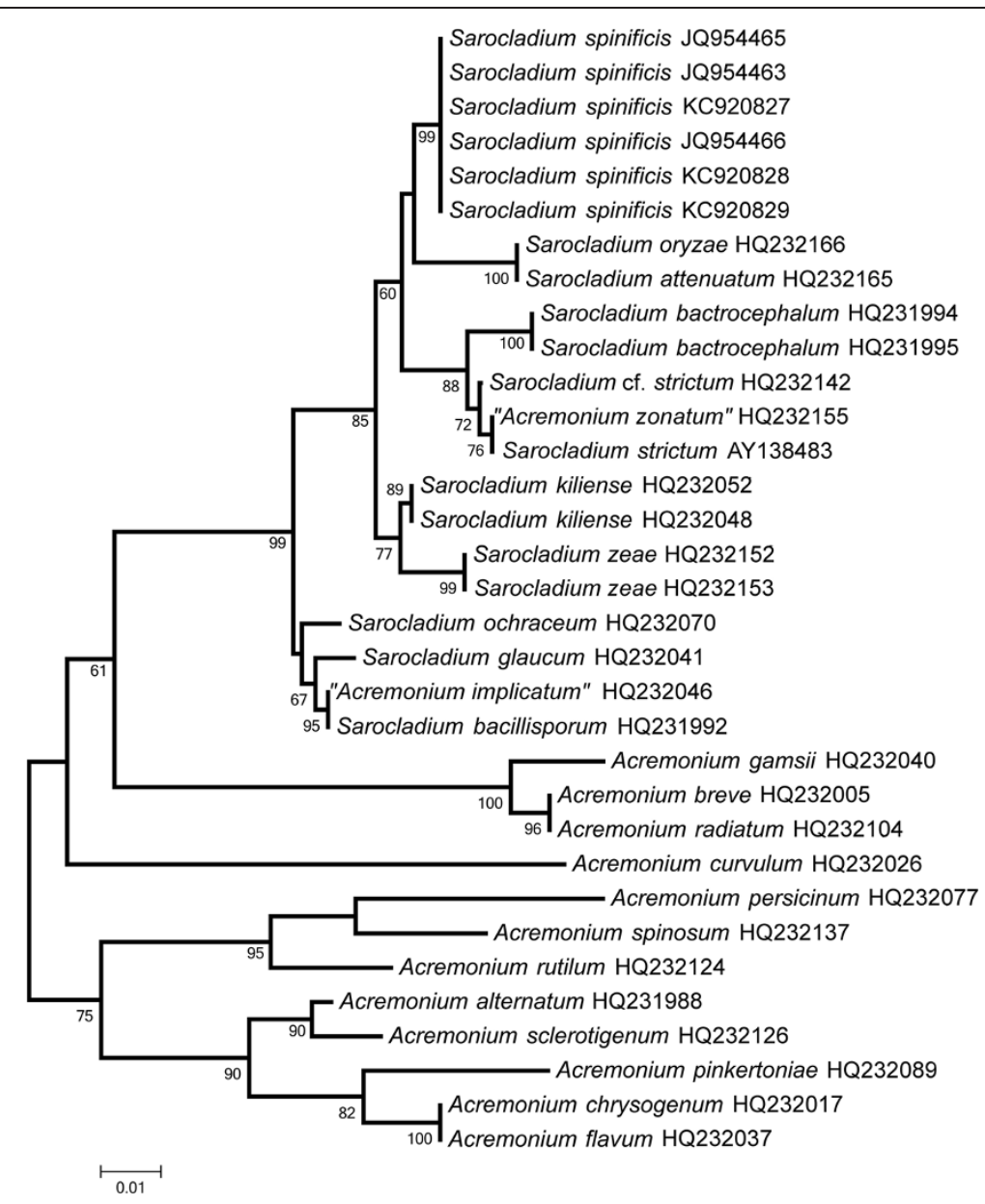

Figure 1 Phylogenetic tree derived from analysis of the LSU rDNA sequences of species of Sarocladium and related Acremonium species using the Maximum Likelihood method by MEGA5. Bootstrap support values above $50 \%$ of 1000 replications given below the branches.

variable species without clear-cut diagnostic characteristics (Domsch et al., 2007).

\section{Taxonomy}

Sarocladium spinificis Yu Hung Yeh \& R. Kirschner, sp. nov. Figure 2(2-6).

MycoBank no.: MB 805250.

\section{Holotypus}

Dried culture from isolate from root fragment of living Spinifex littoreus, Taiwan, Taoyuan County, Kuanyin Township, 27.04.2011, Yu-Hung Yeh Z0106 (TNM).

Ex-type strain: BCRC 34941. LSU rDNA sequence: GenBank JQ954463.

Colonies on malt extract agar $28.5-30 \mathrm{~mm}$ diam. after 10 days at $25^{\circ} \mathrm{C}$ in the dark, flat or at colony centre folded into low wrinkles, overall mycelium appressed forming conspicuous concentric zones. Surface color white (colors Oac909) to pale orange (colors Oac683 and Oac794), reverse orange yellowish (ranging from colors Oac812 to Oac814), sometimes a soluble yellow pigment diffusing into the agar. On CMA, the color is white, on PDA yellow.

Hyphae smooth, hyaline, 1-3 $\mu$ m wide, without swellings or chlamydospores. Conidiophores reduced to conidiogenous cells, not stained with cotton blue. Phialides attached singly to subtending hyphae, $20-37.5(-42) \times 1.5-2 \mu \mathrm{m}$, robust in appearance, mostly straight, subcylindrical, tapering towards the apex up to $0.5-1 \mu \mathrm{m}$, with a small, indistinct, collarette. Adelophialides rare, $3 \times 1 \mu \mathrm{m}$. Conidia formed in slimy heads, cylindrical or ellipsoidal, hyaline, smooth, 5-8 $(-13) \times 1-2 \mu \mathrm{m}(\mathrm{n}=30)$. Conidial widths of five different strains including the ex-type strain (30 conidia measured per strain) were identical $(1-2 \mu \mathrm{m})$, but maximum lengths were slightly different, namely $7-9(-13) \mu \mathrm{m}$.

\section{Etymology}

Referring to the host genus Spinifex.

\section{Teleomorph}

Unknown. 

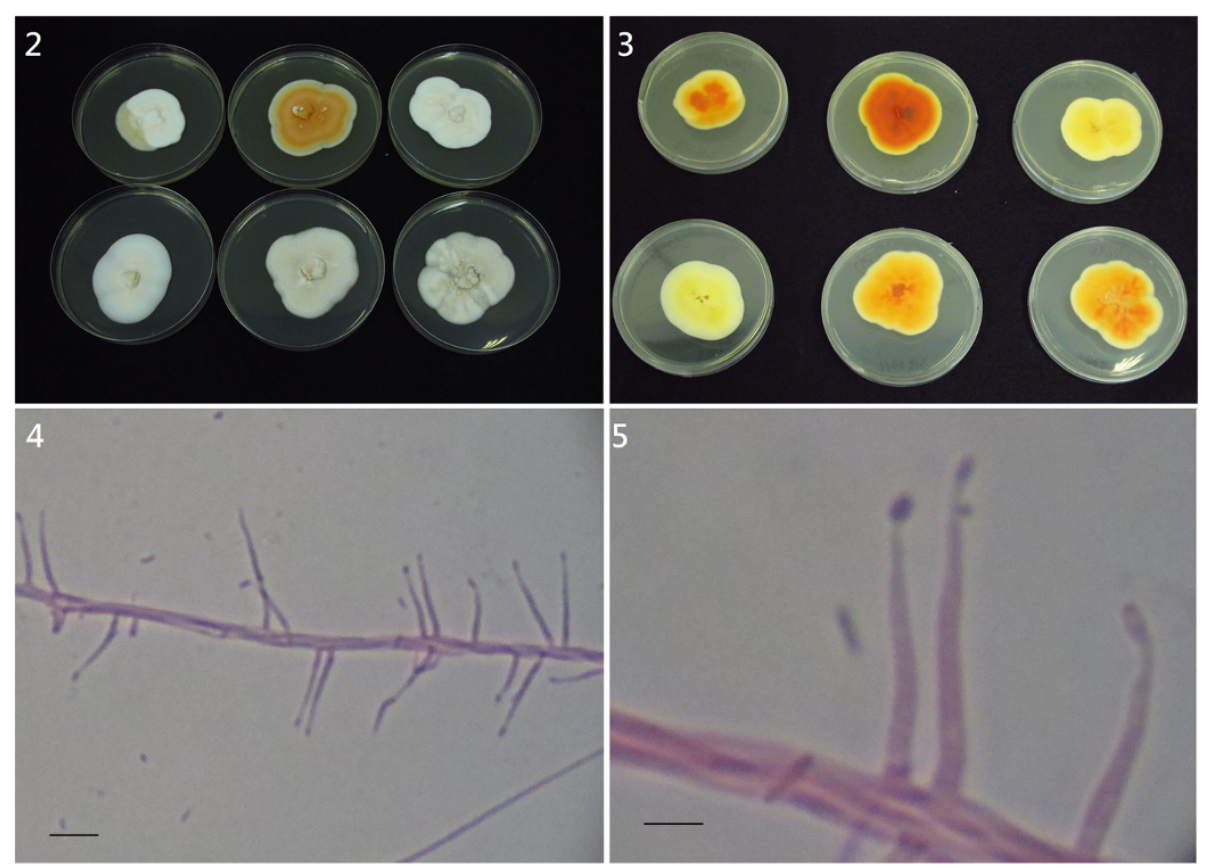

6

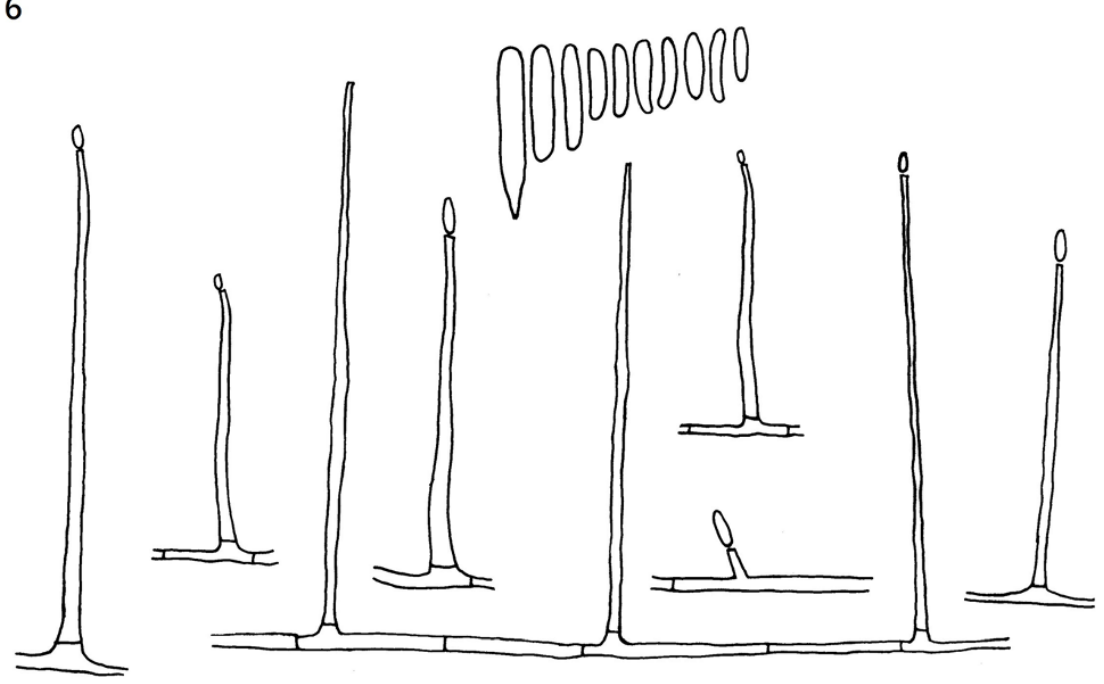

Figure 2 Sarocladium spinificis. 2-3, Culture morphology of six strains; colonies on malt extract agar after 10 days at $25^{\circ} \mathrm{C}$ in the dark (upper isolates from left to right S0101, Z0504, Z1602, lower from left to right Z0106, Z1003, Z1701. $9 \mathrm{~cm}$ Petri dishes); 2, Upper side; 3, Lower side; 4-5, Phialides on hyphae (stained with phloxine); $\mathbf{6}$, Drawing of phialides and conidia. Scale bars: $\mathbf{4}=20 \mu \mathrm{m}, \mathbf{5 - 6}=10 \mu \mathrm{m}$.

\section{Other specimens examined with cultures deposited at BCRC}

All isolated as endophytes from living Spinifex littoreus, Taiwan; Miaoli County, Houlong Township, from root, 01.08.2011, Yu-Hung Yeh Z0504 (BCRC FU30127, LSU rDNA: KF269096; ITS sequence: KF269096); Chiayi County, coastal wetland close to Haomeiliao, from stem, 02.12.2011, Yu-Hung Yeh Z1003 (BCRC FU30128, LSU rDNA: JQ954466); from seed, 02.12.2011, Yu-Hung Yeh S0101 (BCRC FU30126, LSU rDNA: JQ954465); same place, from root, 25.06.2012, Yu-Hung Yeh Z1701 (BCRC FU30130, LSU rDNA: KC920829); Pingtung County, Hengchun Tropical Botanic Garden, 200-300 m above sea level, from stem, 10.06.2012, Yu-Hung Yeh Z1602 (BCRC FU30129, LSU rDNA: KC920828).

\section{Discussion}

The DNA results indicate that the species is to be identified as a member of Sarocladium, which is confirmed by morphology. The species is proposed as new because of 
DNA sequence differences, yellow-orange pigmentation of the cultures, absence of conidiophore branching, and comparatively long conidia. Some strains produce a soluble yellow pigment that diffuses into the agar. After the most recent revision of Acremonium and Sarocladium (Summerbell et al., 2011) the genus hitherto contains 11 species. DNA data are available for nine species (Summerbell et al., 2011; this report). Additionally, the morphological characters of the 11 described species were compiled from the literature: S. attenuatum, S. bacillisporum (Onions \& G.L. Barron) Summerb., S. bactrocephalum (W. Gams) Summerb., S. glaucum (W. Gams) Summerb., S. kiliense, S. mycophilum, S. ochraceum (Onions \& G.L. Barron) Summerb., S. oryzae, S. sinense, S. strictum (W. Gams) Summerb., and S. zeae (Gams and Hawksworth, 1976; Helfer, 1991; Liao and Wu, 2000). None of these species produces a soluble pigment diffusing into the agar. Only six of eleven species form somewhat yellow or orange colonies (S. bacillisporum, $S$. kiliense, S. mycophilum, S. ochraceum, S. strictum, and S. zeae). Among these six species, branched conidiophores were reported for S. kiliense, S. mycophilum, S. strictum and $S$. zeae, whereas branched conidiophores are absent in the new species. Sarocladium strictum is a variable species with few particular characteristics (Domsch et al., 2007). In S. strictum, branched conidiophores are usually absent and colonies pink colored, and conidia are shorter than in all the rest of the species (Gams, 1971; Domsch et al., 2007). It can, however, also include sometimes orange colonies and occasionally branched conidiophores (de Hoog et al., 2000). In some strains, both unusual characteristics (orange colonies and branched conidiophores) are combined together. Such untypical strains of S. strictum could be distinguished only by shorter conidia [3.3-5.5(-7.0) $\mu \mathrm{m}$, Gams, 1971] and molecular data from S. spinificis. Sarocladium bacillisporum and S. ochraceum are the only species with yellow or orange colonies and unbranched conidiophores. Sarocladium bacillisporum differs from $S$. spinificis by its catenate conidia. Sarocladium spinificis differs from $S$. ochraceum by having longer conidia [5-8 $(-13) \times 1-2 \mu \mathrm{m}$ vs. $4.4-5.1 \times 1.3-1.5 \mu \mathrm{m}]$. In $S$. ochraceum, the conidia contribute to the ochre yellow color of dusty dry colonies (Gams, 1971). Summerbell et al. (2011) indicated that Acremonium implicatum (J.C. Gilman \& E. V. Abbott) W. Gams might belong to Sarocladium. This species, however, produces conidia in dry chains (Gams, 1971). For Acremonium species now classified in Sarocladium, Gams (1971) and Domsch et al. (2007) indicated further similar Acremonium species for morphological comparison. From our comparison of S. spinificis with the descriptions of these species, we concluded that S. spinificis is distinct.

Sarocladium now comprises 12 species (if S. attenuatum and S. oryzae are considered separate species), of which eight species are discussed in Summerbell et al. (2011), including seven species described in detail by Gams (1971), but scattered among different species of Acremonium, whereas the descriptions of the other species are given in further publications (Gams and Hawksworth, 1976; Helfer, 1991; Liao and Wu, 2000). We compiled the diagnostic characters of the 12 species from the literature in order to construct following key:

1. Conidiophores almost branched ... 2

1. Conidiophores unbranched ... 3

2. Culture color yellow, becoming brown from the center, on fungi ...

... S. mycophilum Helfer

2. Culture color different from above ... 4

3. Culture color is intensively greyish green ... ... S. glaucum (W. Gams) Summerb.

3. Culture color different from above ... 8

4. Conidia usually up to $7 \mu \mathrm{m}$ long, causing rice sheath blast ... 5

4. Conidia usually up to $6 \mu \mathrm{m}$, not causing disease on rice ... 6

5. Conidium size $4.5-8(-14) \times 0.6-1.0 \mu \mathrm{m} \ldots$ ... S. attenuatum W. Gams \& D. Hawksw.

5. Conidium size $3.5-7 \times 1-1.5 \mu \mathrm{m} \ldots$ ... S. oryzae (Sawada) W. Gams \& D. Hawksw.

5. Conidium size $3.75-11.5 \times 1.75-2.54 \mu \mathrm{m} \ldots$ ... S. sinense J.D. Chen, Guo C. Zhang \& X.H. Fu

6. With chlamydospores, colonies on Sabouraud agar brown ... ... S. kiliense (Grütz) Summerb.

6. Without chlamydospores ... 7

7. Conidiophore often repeatedly branched; tips of phialides with localized wall thickening and frequently wavy in outline beneath this, conidiophore base strongly stained with blue stains ...

...S. zeae (W. Gams \& D.R. Sumner) Summerb.

7. Conidiophores usually consisting of phialides; phialides lacking wall thickening and smooth in outline, in some case somewhat stainable with blue stains...

... S. strictum (W. Gams) Summerb.

8. Conidia usually up to $8 \mu \mathrm{m}$ long, on Spinifex littoreus ... S. spinificis

8. Conidia shorter ... 9

9. Conidia usually in chains ...

... S. bacillisporum (Onions \& G.L. Barron) Summerb.

9. Conidia in slimy drops ... 10

10. Conidia with pointed ends, colonies dry and dusty ... ... S. ochraceum (Onions \& G.L. Barron) Summerb.

10. Not as above ... 11

11. Conidia $<1 \mu \mathrm{m}$ wide ... S. bactrocephalum (W. Gams) Summerb.

11. Conidia $\geq 1 \mu \mathrm{m}$ wide ... S. strictum (W. Gams) Summerb. 
Possibly the three species Sarocladium attenuatum, S. oryzae, and $S$. sinense are the same species. A possible synonymy of $S$. attenuatum and $S$. oryzae was proposed by Bridge et al. (1989) based on biochemical and morphometric analyses and further supported because of identical DNA sequences (Bills et al., 2004). The ITS sequence obtained from a culture of S. attenuatum, however, by Summerbell et al. (2011) was different from that obtained from the same culture by Bills et al. (2004). We did not find morphological differences in the description of S. sinense by Liao and Wu (2000) from descriptions of S. oryzae (Gams and Hawksworth, 1976). These three species all cause sheath disease in Oryza sativa and have no significant difference in morphology.

Sarocladium spp. are often associated with Poaceae, such as S. attenuatum, S. oryzae, and S. sinense in rice. Sarocladium zeae is known as a protective maize endophyte (Wicklow et al., 2005; Poling et al., 2008). Sarocladium bacillisporum, S. bactrocephalum and S. strictum also have been recorded as endophytes from Poaceae (Tunali et al., 2000; Hormazabal and Piontelli, 2009). By molecular techniques, the most abundant clones isolated from hypersaline microbial mats revealed to belong to $S$. strictum (as Acremonium strictum, Cantrell et al., 2013), indicating that Sarocladium species might have the ability to grow in environments with high salinity. Since S. spinificis has been discovered as grass endophyte from a marine coastal habitat, we hypothesize that this species has a similar function as other endophytes conferring salt tolerance to coastal plants (Rodriguez et al., 2008).

\section{Conclusions}

Sarocladium spinificis isolated as endophyte from the coastal plant Spinifex littoreus is proposed as a new species. LSU rDNA sequences in addition to conidiophore branching and conidium size are useful characters for distinguishing between species of Sarocladium.

\section{Competing interests}

The authors declare that they have no competing interests.

\section{Authors' contributions}

YHY collected the materials, carried out the experiments, morphological and phylogenetical study, drafted the manuscript. RK supervised the study, refined the taxonomic treatment and refined the text with respect to language and the literature citations. Both authors read and approved the final version of manuscript.

\section{Acknowledgements}

We thank I.-S. Lee (Academia Sinica) for help during collection in Taiwan and S.-H. Wu (Hengchun Tropical Botanic Garden) for kindly arranging collection at Hengchun Tropical Botanic Garden, Taiwan. K.-L. Pang is thanked for his tip about a collection site. This study was supported by the National Science Council of Taiwan (NSC100-2621-B-008-001-MY3).

Received: 10 September 2013 Accepted: 31 December 2013

Published: 5 February 2014

\section{References}

Bills GF, Platas G, Gams W (2004) Conspecificity of the cerulenin and helvolic acid producing 'Cephalosporium caerulens', and the hypocrealean fungus Sarocladium oryzae. Mycol Res 108:1291-1300

Bridge PD, Hawksworth DL, Kavishe DF, Farnell PA (1989) A revision of the species concept in Sarocladium, the causal agent of sheath-rot in rice and bamboo blight, based on biochemical and morphometric analyses. Plant Pathol 38:239-245

Cantrell SA, Tkavc R, Gunde-Cimerman N, Zalar P, Acevedo M, Báez-Félix C (2013) Fungal communities of young and mature hypersaline microbial mats. Mycologia 105:827-836

De Hoog GS, Guarro J, Gene J, Figueras MJ (2000) Atlas of clinical fungi, 2nd edn. Centraalbureau voor Schimmelcultures, Utrecht, The Netherlands

Domsch KH, Gams W, Anderson T-H (2007) Compendium of soil fungi, 2nd edn. IHW-Verlag, Eching

Gams W (1971) Cephalosporium-artige Schimmelpilze (Hyphomycetes) G. Fischer, Stuttgart

Gams W, Hawksworth DL (1976) ['1975'] The identity of Acrocylindrium oryzae Sawada and a similar fungus causing sheath rot of rice. Kavaka 3:57-61

Gardes M, Bruns TD (1993) ITS primers with enhanced specificity for Basidiomycetes: application to identification of mycorrhizae and rusts. Mol Ecol 2:113-118

Helfer W (1991) Pilze auf Pilzfruchtkörpern: Untersuchungen zur Ökologie. Systematik und Chemie, IHW-Verlag, Eching

Hormazabal E, Piontelli E (2009) Endophytic fungi from Chilean native gymnosperms: antimicrobial activity against human and phytopathogenic fungi. World J Microb Biot 25:813-819

Liao Y, Wu B (2000) A study on cause of rice purple sheath disease. Acta Agric Univ Jiangxiensis 22:62-67

Maun MA (2009) The biology of coastal sand dunes. Oxford University Press, New York

Poling SM, Wicklow DT, Rogers KD, Gloer JB (2008) Acremonium zeae, a protective endophyte of maize, produces dihydroresorcylide and 7hydroxydihydroresorcylides. J Agric Food Chem 56:3006-3009

Racedo J, Salazar SM, Castagnaro AP, Díaz Ricci JC (2013) A strawberry disease caused by Acremonium strictum. Eur J Plant Pathol 137:649-654

Rodriguez RJ, Henson J, Van Volkenburgh E, Hoy M, Wright L, Beckwith F, Kim YO, Redman RS (2008) Stress tolerance in plants via habitat-adapted symbiosis. ISME J 2:404-416

Saleh AA, Leslie JF (2004) Cephalosporium maydis is a distinct species in the Gaeumannomyces-Harpophora species complex. Mycologia 96:1294-1305

Summerbell RC, Gueidan C, Schroers H-J, de Hoog GS, Starink M, Arocha Rosete Y, Guarro J, Scott JA (2011) Acremonium phylogenetic overview and revision of Gliomastix, Sarocladium, and Trichothecium. Stud Mycol 68:139-162

Tamura K, Peterson D, Peterson N, Stecher G, Nei M, Kumar S (2011) MEGA5 molecular evolutionary genetics analysis using maximum likelihood, evolutionary distance, and maximum parsimony methods. Mol Biol Evol 28:2731-2739

Tschen JS-M, Chen L-L, Hsieh S-T, Wu T-S (1997) Isolation and phytotoxic effect of helvolic acid from plant pathogenic fungus Sarocladium oryzae. Bot Bull Acad Sin 38:251-256

Tunali B, Shelby RA, Morgan-Jones G, Kodan M (2000) Endophytic fungi and ergot alkaloids in native Turkish grasses. Phytoparasitica 28:375-377

White TJ, Bruns TD, Lee S, Taylor J (1990) Amplification and direct sequencing of fungal ribosomal RNA genes for phylogenetics. In: Innis MA, Gelfand DH, Sninsky JJ, White TJ (eds) PCR protocols, a guide to methods and applications. Academic Press, San Diego, California, pp 315-322

Wicklow DT, Roth S, Deyrup ST, Gloer JB (2005) A protective endophyte of maize: Acremonium zeae antibiotics inhibitory to Aspergillus flavus and Fusarium verticillioides. Mycol Res 109:610-618

doi:10.1186/1999-3110-55-25

Cite this article as: Yeh and Kirschner: Sarocladium spinificis, a new endophytic species from the coastal grass Spinifex littoreus in Taiwan. Botanical Studies 2014 55:25. 